\title{
Vergleich der Raumgliederung Europas mit bestehenden Raumgliederungen - Teil 3
}

\author{
Inga Weustermann · Roland Pesch • Gunther Schmidt · Winfried Schröder
}

Erhalten: 21. Juli 2008/Akzeptiert: 25. Februar 2009/Online veröffentlicht: 25. März 2009

(C) Springer-Verlag 2009

Zusammenfassung Hintergrund und Ziel In den ersten beiden Beiträgen der Beitragsserie Ökologische Raumgliederung Europas (Hornsmann et al. 2008; Weustermann et al. 2009) wurde die Methodik zur Ableitung der Raumgliederung für Europa (ELCE - die in diesem Beitrag verwendeten Abkürzungen sind in Tabelle 1 zusammengetragen) vorgestellt und einzelne Raumklassen beispielhaft beschrieben. In diesem dritten Artikel der Serie wird die in unterschiedlicher Gliederungstiefe vorliegende ELCE zwei Klassifikationen Europas gegenübergestellt: der Digital Map of European Ecological Regions (DMEER) sowie der Environmental Stratification of Europe (EnS). Ergänzend dazu wurde die ELCE mit der für Deutschland berechneten ökologischen Raumgliederung verglichen. Der Abgleich erfolgte sowohl fachlich als auch statistisch und diente der Überprüfung der fachlichen Konsistenz der ELCE.

Material und Methoden Die Raumgliederungen werden hinsichtlich der Eingangsdaten, des Verfahrens und des Verwendungszwecks vorgestellt. Die ELCE wurde mit den drei ausgewählten Gliederungen im GIS verschnitten und anschließend

Serienherausgeber: Gunther Schmidt · Winfried Schröder Vorwort (Schmidt und Schröder 2008)

Teil I: Berechnung einer landschaftsökologischen Raumgliederung Europas (Hornsmann et al. 2008)

Teil II: Beschreibung der landschaftsökologischen Raumgliederung Europas (Weustermann et al. 2009)

Teil III: Vergleich der landschaftsökologischen Raumgliederung Europas mit bestehenden Raumgliederungen

Teil IV: Repräsentanzanalyse für ökologische Langzeitmessprogramme in Europa

Beitragsherausgeber: Henner Hollert

I. Weustermann $(\bowtie) \cdot$ R. Pesch · G. Schmidt · W. Schröder Lehrstuhl für Landschaftsökologie, Hochschule Vechta,

PF 1553, 49364 Vechta, Deutschland

E-Mail: iweustermann@iuw.uni-vechta.de im Hinblick auf die Flächengröße der einzelnen Teilpolygone kreuztabelliert. Hierbei wurde jeweils der prozentuale Anteil der Raumklassen der zu vergleichenden Raumgliederung an den Raumklassen der ELCE (EC) bestimmt und umgekehrt. Die Ermittlung der Ähnlichkeit zwischen den Raumklassen erfolgte über geeignete statistische Maßzahlen.

Ergebnisse Es zeigen sich hohe Übereinstimmungsraten der ELCE mit den Vergleichsraumgliederungen für Europa. Es ergibt sich eine mittlere Abdeckung der EC durch die Raumklassen der DMEER von $84 \%$ bzw. der DMEER-Klassen durch die EC von $69 \%$. Zwischen den EC und den Strata der EnS beträgt der Wert 67 bzw. 72\%. Zudem ist die ELCE in der Lage, die wesentlichen ökologischen Gegebenheiten Deutschlands wiederzugeben. Der Wert für die mittlere Überdeckung der EC und der Klassen der Ökogliederung Deutschlands liegt bei 75 bzw. $80 \%$.

Diskussion Die DMEER-Klassen decken die EC deshalb deutlich besser ab als umgekehrt, weil größere DMEERKlassen die im Schnitt deutlich kleineren EC z.T. vollständig überlagern. Der Hauptunterschied zwischen der ELCE und der EnS ist durch das methodische Vorgehen bei der Ableitung der Gliederungen begründet. Die EnS ist in Südeuropa differenzierter ausgegliedert, während die ELCE in Mitteleuropa kleinere, stärker unterteilte Raumklassen aufweist. Die guten Übereinstimmungen zwischen der Deutschland-Gliederung und der ELCE deuten darauf hin, dass die ELCE im europäischen Maßstab die ökologischen Strukturen der einzelnen Länder gut wiedergibt.

Schlussfolgerungen Basierend auf den ausgewählten europäischen Raumgliederungen kann die ELCE als fachlich plausibel und als geeignete Ergänzung zu diesen eingestuft werden. Sie kann jedoch kein Ersatz für nationale Gliederungen sein, die auf höher aufgelösten Daten basieren. Für europaweite Repräsentanzanalysen von Messnetzen stellt die ELCE eine geeignete Grundlage dar. 
Empfehlungen In Ergänzung zu der landschaftsökologischen Raumgliederung Deutschlands sollte die ELCE in zukünftigen Arbeiten auch mit anderen nationalen Raumgliederungen verglichen werden. Eine Neuberechnung der ELCE sollte vorgenommen werden, wenn ein geeignetes Verfahren zur Verfügung steht, das eine höhere Anzahl an Ausprägungen der Zielvariablen zulässt und auf höher aufgelöste Eingangsdaten zurückgreifen kann. Zudem wäre es im Hinblick auf die klimatischen Veränderungen in den letzten 20 Jahren von Vorteil, aktuellere Klimadaten für die Ableitung zu verwenden.

Ausblick Die fachliche Konsistenz der ELCE wird durch die dargestellten Berechnungen untermauert. Ein vierter Artikel soll daher Anwendungsmöglichkeiten der ELCE im Hinblick auf die Repräsentanz europäischer Umweltmessnetze behandeln.

Schlüsselwörter Classification and Regression Trees (CART) - Digital Map of European Ecological Regions (DMEER) · Europäische Raumgliederung (ELCE) · Klassifizierung · Ökologische Raumgliederung · Ökologische Raumgliederung Deutschlands · Repräsentanz · Environmental Stratification of Europe $($ EnS) $\cdot$ Umweltbeobachtung $\cdot$ Umweltmonitoring

\section{Comparison of the ecological land classification of Europe with existing land classifications}

\begin{abstract}
Background, aim and scope The first two articles of this series (Hornsmann et al. 2008; Weustermann et al. 2009) comprised how the Ecological Land Classification of Europe (ELCE) was calculated. Moreover several ecological classes were described exemplarily. In this part 3 of the series the ELCE is compared with two European ecoregionalisations at several levels of differentiation. These are the Digital Map of European ecological regions (DMEER) and the Environmental Stratification of Europe (EnS). In addition the ELCE was opposed to the ecoregionalisation of Germany. The alignment was carried out both functionally and statistically and served as a basis for the examination of the functional consistency of the ELCE.

Materials and methods The ecological land classifications were described concerning their input data, the method of derivation and the intended use. The ELCE and the selected three ecoregionalisations were intersected in a GIS and crosstabulated with regard to the acreage of each polygon. These cross tabulations were performed by listing the percentage of ecoregions of e. g. DMEER to the ecological classes of the ELCE (EC) and vice versa. The similarity of the compared ecoregions was estimated by means of statistics.

Results The comparisons show high rates of similarity of the ELCE to the European land classifications. The mean
\end{abstract}

overlap of the EC with the classes of the DMEER is 84 and $69 \%$ vice versa. The EC are covered by the strata of the EnS with 67 and $72 \%$, respectively. In Germany, ELCE reflects the most important ecological conditions. The mean overlap of the ecoregions in this case is 75 and $80 \%$ vice versa.

Discussion The classes of the DMEER show a better overlap of the EC as vice versa due to their larger spatial extent. The main difference between the ELCE and the EnS is based on the procedure of the derivation of the regionalisations. While the classes of the EnS are more differentiated in the South of Europe, the ecoregions of ELCE are smaller and more subdivided in the middle of Europe. The high overlap between the ELCE and the ecoregionalisation of Germany can be indicative for the good account of the ecological structures of the ELCE in the European dimension.

Conclusions The ELCE is functionally plausible and a useful complement to the existing land classifications of Europe. Nevertheless, it cannot substitute land classifications on the national level calculated with more detailed input data. The ELCE can be used testing the representativity of environmental monitoring networks in Europe.

Recommendations In future, the ELCE should be compared with other national landscape classifications. A new calculation of ELCE would only be useful after the development of a highly powerful derivation method and on the basis of more detailed input data. Moreover, due to the observed climate change in the past 20 years, up-to-date data on climate should be used for the calculations.

Perspectives The ELCE could be proven to be functionally consistent. Therefore the fourth article will focus on the investigation of the landscape representativity of environmental monitoring networks on the basis of the ELCE.

Keywords Classification - Classification and Regression Trees (CART) · Digital Map of European Ecological Regions (DMEER) · Ecological Landscape Classification of Europe (ELCE) · Ecological research · Ecoregionalisation - Ecoregionalisation of Germany · Environmental monitoring · Environmental Stratification of Europe (EnS) · Representativity

\section{Hintergrund und Ziel}

Mit dem Klassifikationsverfahren Classification and Regression Trees (CART) wurden in einem von der Deutschen Forschungsgemeinschaft (DFG) geförderten Projekt landschaftsökologische Raumgliederungen für Europa (Ecological Landscape Classification of Europe, ELCE) in zwölf verschiedenen Differenzierungsstufen $(40,50,60,80,100$, $120,125,140,150,160,180,200)$ berechnet (Hornsmann et al. 2008, Weustermann et al. 2009). Derartige auf statistischen Verfahren basierende landschaftsökologische Raum- 
Tabelle 1 Abkürzungsverzeichnis

\begin{tabular}{|c|c|}
\hline Abkürzung & Bedeutung \\
\hline $\mathrm{BfN}$ & Bundesamt für Naturschutz \\
\hline CART & $\begin{array}{l}\text { Classification and Regression Trees, Klassifika- } \\
\text { tions- und Regressionsbäume }\end{array}$ \\
\hline CRU & $\begin{array}{l}\text { Climatic Research Unit, Klimaforschungs- } \\
\text { Referat }\end{array}$ \\
\hline DER & $\begin{array}{l}\text { Digital ecological regions, Digitale Ökoregionen } \\
\text { der DMEER }\end{array}$ \\
\hline DMEER & $\begin{array}{l}\text { Digital Map of European Ecological Regions, } \\
\text { digitale Karte der europäischen Ökoregionen }\end{array}$ \\
\hline $\mathrm{EC}$ & $\begin{array}{l}\text { Ecological class, Ökologische Raumklasse der } \\
\text { ELCE }\end{array}$ \\
\hline EEA & $\begin{array}{l}\text { European Environment Agency, Europäische } \\
\text { Umweltagentur }\end{array}$ \\
\hline ELCE & $\begin{array}{l}\text { Ecological land classification of Europe, Ökolo- } \\
\text { gische Raumgliederung Europas }\end{array}$ \\
\hline $\begin{array}{l}\text { ELCE40, ELCE50 } \\
\text { usw. }\end{array}$ & $\begin{array}{l}\text { Ökologische Raumgliederung mit 40, } 50 \text { etc. } \\
\text { Endklassen }\end{array}$ \\
\hline EnS & $\begin{array}{l}\text { Environmental stratification, Stratification of } \\
\text { Europe, Einteilung von Europa }\end{array}$ \\
\hline $\mathrm{ETC} / \mathrm{NC}$ & $\begin{array}{l}\text { European Topic Center on Nature Conservation/ } \\
\text { Naturschutz }\end{array}$ \\
\hline FAO & $\begin{array}{l}\text { Food and Agriculture Organization, } \\
\text { Ernährungs- und Landwirtschaftsorganisation } \\
\text { der Vereinten Nationen }\end{array}$ \\
\hline ISODATA & $\begin{array}{l}\text { Iterative Self-Organizing Data Analysis Tech- } \\
\text { nique, Iterative Selbstorganisierende Analyse- } \\
\text { technik }\end{array}$ \\
\hline ITE & Institute of Terrestrial Ecology, Ökologieinstitut \\
\hline ÖK & $\begin{array}{l}\text { Ökoklasse der ökologischen Raumgliederung für } \\
\text { Deutschland }\end{array}$ \\
\hline $\mathrm{PNV}$ & Potenziell natürliche Vegetation \\
\hline PNV-Ke & PNV-Kartierungseinheit \\
\hline
\end{tabular}

gliederungen müssen sowohl inhaltlich als auch statistisch bewertet werden. Hinsichtlich der statistischen Bewertung ist dabei insbesondere die Trennschärfe der landschaftsökologischen Raumklassen zu überprüfen (Schröder et al.
2006). Für ELCE40 erfolgte eine derartige Analyse bereits für die metrisch skalierten Eingangsvariablen zur Höhe und zum Klima anhand inferenzstatistischer Verfahren (Weustermann et al. 2009). Zusätzlich wurden am Beispiel dreier Raumklassen die Unterschiede der landschaftsökologischen Gegebenheiten im Hinblick auf die gesamteuropäischen Verhältnisse aufgezeigt. In Ergänzung zu dieser rein statistischen Bewertung ist es sinnvoll, die ELCE mit anderen anerkannten europäischen Naturraumgliederungen zu vergleichen. In dem vorliegenden dritten Artikel soll die ELCE daher der Digital Map of European Ecological Regions (DMEER) (Painho und Augusto 2005) sowie der Environmental Stratification of Europe (EnS) (Metzger et al. 2005) fachlich und statistisch gegenübergestellt werden. Ferner soll die ELCE mit der für Deutschland berechneten landschaftsökologischen Raumgliederung nach Schröder et al. (2006) abgeglichen werden. Über den Grad der Übereinstimmung der ELCE mit fachlich anerkannten, etablierten Raumgliederungen kann deren fachliche Plausibilität eingeschätzt werden.

\section{Material und Methoden}

\subsection{Material}

Im Folgenden werden die oben genannten Gliederungen hinsichtlich der verwendeten Datengrundlage und des Ableitungsverfahrens vorgestellt. Tabelle 2 zeigt eine Übersicht über die digitalen Raumgliederungen inkl. Angaben zur Datenquelle, zum Stand und zur Auflösung.

ELCE Die Berechnungsgrundlage für die ELCE bilden 48 digitale Karten zur potenziell natürlichen Vegetation (PNV) (Bohn et al. 2000, 2003) im Maßstab 1:2.500.000, zur Bodenart (FAO 1996) im Maßstab 1:5.000.000, zur Höhe über NN in einem Raster von 30 Bogensekunden (ca. $1 \mathrm{~km} \times 1 \mathrm{~km}$ ) (Hastings et al. 1999) sowie Karten zur

Tabelle 2 ELCE und die Vergleichsraumgliederungen

\begin{tabular}{|c|c|c|c|c|c|c|}
\hline Karte, Region & Quelle & Stand & $\begin{array}{l}\text { Auflösung/ } \\
\text { Maßstab }\end{array}$ & Region & $\begin{array}{l}\text { Berechnungs- } \\
\text { verfahren }\end{array}$ & $\begin{array}{l}\text { Anzahl der Ein- } \\
\text { gangs-variablen-/ } \\
\text { Karten }\end{array}$ \\
\hline $\begin{array}{l}\text { Ecological Land Classification of } \\
\text { Europe (ELCE; Ökologische Raum- } \\
\text { gliederung von Europa) }\end{array}$ & $\begin{array}{l}\text { Hochschule Vechta, } \\
\text { LLÖK (Hornsmann } \\
\text { et al. 2008) }\end{array}$ & 2006 & $10 \times 10 \mathrm{~km}^{2}$ & Europa & CART & 48 \\
\hline $\begin{array}{l}\text { Digital map of European Ecological } \\
\text { Regions (DMEER; Digitale Karte der } \\
\text { europäischen Ökoregionen) }\end{array}$ & $\begin{array}{l}\text { EEA; Ersteller: ETC/ } \\
\text { NC (Painho und Au- } \\
\text { gusto 2005) }\end{array}$ & 2000 & $1: 2.500 .000$ & Europa & $\begin{array}{l}\text { Herachisches } \\
\text { Clustering }\end{array}$ & 2 \\
\hline $\begin{array}{l}\text { Stratification of Europe (Einteilung } \\
\text { von Europa) }\end{array}$ & Metzger et al. (2005) & 2005 & $1 \times 1 \mathrm{~km}^{2}$ & Europa & $\begin{array}{l}\text { Hauptkompo- } \\
\text { nentenanalyse, } \\
\text { ISODATA, }\end{array}$ & 20 \\
\hline $\begin{array}{l}\text { Ökologische Raumgliederung } \\
\text { Deutschlands }\end{array}$ & Schröder et al. (2006) & 2002 & $2 \times 2 \mathrm{~km}^{2}$ & Deutschland & CART & 48 \\
\hline
\end{tabular}


räumlichen Struktur von Temperatur, Niederschlag, relativer Luftfeuchtigkeit und Sonnenscheindauer in einem Raster von 10 Bogenminuten (ca. $19 \mathrm{~km} \times 19 \mathrm{~km})(C R U$ 2002, New et al. 2002). Die ELCE soll insbesondere als räumliche Bezugsgrundlage für die Überprüfung der Landschaftsrepräsentanz von Messnetzen der ökologischen Langzeitbeobachtung (Schmidt et al. 2009) sein. Ausführliche Darstellungen der Datengrundlage, Datenaufbereitung und zum CART-Verfahren sowie einige beispielhafte Beschreibungen ausgewählter Raumklassen (Ecological Classes, EC) der ELCE40 finden sich in Hornsmann et al. (2008) und Weustermann et al. (2009).

DMEER Die DMEER im Maßstab $1: 2.500 .000$ wurde vom European Topic Center on Nature Conservation (ETC/NC) unter Beteiligung des Bundesamts für Naturschutz (BfN) zwischen 1995 bis 2000 erstellt (Painho und Augusto 2005). Diese europaweite Ökogliederung ist in 69 Klassen aufgeteilt, die durch Informationen zur PNV und zum Klima beschrieben sind. DMEER sollte als weithin akzeptierte Grundlage für Naturschutzaufgaben z. B. für Berichte und Bewertungen im Rahmen der Flora-Fauna-Habitat-Richtlinie sowie für Bewertungen der Biodiversität auf europäischer Ebene Verwendung finden (EEA 2004, ETC/NC 1997, Painho und Augusto 2005). DMEER wurde unter Verwendung folgender Datengrundlagen berechnet:

- der Karte „The European Land“ des Institute of Terrestrial Ecology (ITE) mit Informationen zu Klima und zur Topografie mit einer Auflösung von $0,5^{\circ} \times 0,5^{\circ}\left(56 \times 56 \mathrm{~km}^{2}\right)$ (Bunce 1995) sowie

- der Karte der PNV Europas des BfN (Stand 1994) im Maßstab 1:2.500.000 (Bohn et al. 2000, 2003).

Die Karte des ITE stellt die Vorgängerversion zur Stratification of Europe (EnS) von Metzger et al. (2005) dar (s. u.). Die PNV-Karte für Europa gleicht der für die Ableitung der ELCE verwendeten PNV. Zur Berechnung der DMEER wurden für alle vorhandenen PNV-Kategorien die Flächenanteile der Raumklassen nach Bunce (1995) ermittelt. Anschließend erfolgte anhand letzterer eine auf hierarchischen Clusterverfahren basierende Zusammenlegung der PNVKlassen. Da die derart berechnete Gliederung Europas keine fachlich befriedigende Lösung darstellte, wurde die Karte auf Grundlage biogeografischen und vegetationskundlichen Fachwissens nachträglich verändert (Painho und Augusto 2005). Die Namensgebung der Digital Ecological Regions (DER) wurde bei der DMEER in Anlehnung an die PNVKarte vorgenommen (Tabelle 3 ).

EnS Ziel der Studie von Metzger et al. (2005) war es, eine statistisch basierte ökologische Gliederung Europas vorzunehmen, die als Grundlage für die Auswahl repräsentativer Probennahmestandorte und als Bezugsgrundlage für Modellierungen und Berichterstattungen geeignet ist. Ihre Verwendbarkeit im Hinblick auf Fragen des Biodiversitäts- und Habitatsmonitorings sowie für Landnutzungs- und Landbedeckungsanalysen wurde bereits getestet (Jongman et al. 2006). Die Ausdehnung der EnS umfasst Mitteleuropa, Nordafrika und den westlichen Teil der Türkei. Angelehnt an die Klassifikation des ITE von Bunce (1995) fanden 20 Variablen als Datengrundlage Verwendung (Metzger et al. 2005): Höhe und Hangneigung (HYDRO1k) des United States Geological Survey (30 Bogensekunden, ca. $1 \mathrm{~km} \times 1 \mathrm{~km})($ USGS 2008); Breitengrad, Ozeanität (Spannweite der Temperatur zwischen Januar und Juli dividiert durch den Sinus der geografischen Breite) und Minimum/ Maximum der Temperatur; Niederschlag sowie prozentuale Sonnenscheindauer jeweils in den Monaten Januar, April, Juli und Oktober (CRU_TS1.2) mit einer Auflösung von $10 \times 10$ Bogenminuten $($ ca. $19 \mathrm{~km} \times 19 \mathrm{~km})($ CRU 2008; Mitchell et al. 2004). Da die verwendeten Klimadaten den Osten Europas sowie Island aussparen, bestimmen diese die Ausdehnung der EnS. Der entsprechende Datensatz besteht aus Zeitreihen von Klimadaten und umfasst eine Zeitspanne von 1901 bis 2000. Die Klimadaten wurden von Metzger et al. (2005) mittels bilinearer Interpolation in die für die EnS gewählte Auflösung von $1 \mathrm{~km} \times 1 \mathrm{~km}$ umgerechnet.

Die Berechnung der EnS fußt auf folgendem methodischen Prinzip: In einem ersten Schritt erfolgte mithilfe einer Hauptkomponentenanalyse eine Verringerung der redundanten Informationen in den 20 Ausgangsvariablen in drei Hauptkomponenten, die $88 \%$ der Gesamtvarianz abdecken. Anschließend wurden die Hauptkomponenten mit dem partitionierenden Iterative-Self-Organising-DataAnalysis-Verfahren (ISODATA) geclustert. Getrennt nach Nordeuropa (70\% von Europa) und Südeuropa (30\%) wurde das Gebiet in insgesamt 70 EnS-Strata (40 in Nordeuropa, 30 in Südeuropa) eingeteilt. In der Nachbearbeitung wurden kleine Gebiete mit weniger als $250 \mathrm{~km}^{2}$ dem Stratum der benachbarten Rasterzellen zugeordnet. Aus Raumklassen, deren Abschnitte räumlich getrennt voneinander auftraten (z. B. am Atlantik und an der Adria), wurden mehrere Raumklassen gebildet, da die Gebiete laut Metzger et al. (2005) zwar klimatisch ähnlich seien, sich jedoch insbesondere in der Artenzusammensetzung deutlich unterscheiden würden. Die resultierenden 84 Strata wurden abschließend zu 13 Großeinheiten zusammengefasst. Die Benennung dieser Großeinheiten erfolgte auf Basis der wesentlichen klimatischen Merkmale und/oder der geografischen Lage. Die Bezeichnung der 84 Strata setzt sich aus der Abkürzung dieser übergeordneten Regionen und einer fortlaufenden Nummer zusammen (Tabelle 4). Die Großeinheiten der EnS fanden Verwendung bei der Ableitung der Europäischen Landschaftscharaktergebiete (European Landscape Character Areas) von Wascher (2005), aus der die sogenannte LANMAP 2 (European Landscape Map) im Maßstab 1:2.000.000 resultierte 
Tabelle 3 Legende zu den Raumklassen der Digital Map of European Ecological Regions (DMEER)

\begin{tabular}{|c|c|c|c|}
\hline ID_Nr & Ecoregion & ID_Nr & Ecoregion \\
\hline 10 & Appenine deciduous montane forests & 97 & Faroe Islands boreal grasslands \\
\hline 11 & Southern Temperate Atlantic & 100 & Kazakh steppe \\
\hline 12 & Balkan mixed forests & 102 & Middle East steppe \\
\hline 13 & Baltic mixed forests & 104 & Pontic steppe \\
\hline 14 & Cantabrian mixed forests & 138 & Arctic desert \\
\hline 15 & Caspian Hyrcanian mixed forests & 143 & Kola Peninsula tundra \\
\hline 16 & Caucasus mixed forests & 145 & Northwest Russian/Novaya Zemlya tundra \\
\hline 17 & Celtic broadleaf forests & 147 & Scandinavian montane birch forest and grasslands \\
\hline 18 & Central Anatolian deciduous forests & 151 & Yamalagydanskaja tundra \\
\hline 20 & Central European mixed forests & 152 & Aegean \& West Turkey sclerophyllous and mixed forest \\
\hline 25 & Crimean submediterranean forest complex & 153 & Anatolian conifer and deciduous mixed forests \\
\hline 27 & Dinaric Mountains mixed forests & 154 & Corsican montane broadleaf and mixed forests \\
\hline 28 & East European forest steppe & 155 & Crete Mediterranean forests \\
\hline 29 & Eastern Anatolian deciduous forests & 156 & Cyprus Mediterranean forests \\
\hline 31 & English Lowlands beech forests & 157 & Eastern Mediterranean coniferous/sclerophyllous/broadleaf forests \\
\hline 32 & Euxine-Colchic deciduous forest & 158 & Iberian conifer forests \\
\hline 38 & North Atlantic moist mixed forests & 159 & Iberian sclerophyllous and semi-deciduous forests \\
\hline 41 & Pannonian mixed forests & 160 & Illyrian deciduous forests \\
\hline 42 & Po Basin mixed forests & 161 & Italian sclerophyllous and semi-deciduous forests \\
\hline 43 & Pyrenees conifer and mixed forests & 162 & Northeastern Spain \& Southern France Mediterranean \\
\hline 45 & Rodope montane mixed forests & 163 & Northwest Iberian montane forests \\
\hline 46 & Sarmatic mixed forests & 164 & Pindus Mountains mixed forests \\
\hline 55 & Western European broadleaf forests & 165 & South Appenine mixed montane forests \\
\hline 56 & Alps conifer and mixed forests & 166 & Southeastern Iberian shrubs and woodlands \\
\hline 58 & Caledon coniferous forests & 167 & Southern Anatolian montane conifer and deciduous forests \\
\hline 59 & Carpathian montane coniferous forests & 168 & Southwest Iberian Mediterranean sclerophyllous and mixed forests \\
\hline 63 & Elburz Range forest steppe & 169 & Tyrrhenian-Adriatic sclerophyllous and mixed forests \\
\hline 70 & Northern Anatolian conifer and deciduous forests & 172 & Arabian desert and East Sahero-Arabian xeric shrub \\
\hline 75 & Scandinavian coastal coniferous forests & 174 & Azerbaijan shrub desert and steppe \\
\hline 80 & Iceland boreal birch forest and alpine tundra & 177 & Caspian Lowland desert \\
\hline 86 & Scandinavian and Russian taiga & 188 & Kazakh semi-desert \\
\hline 88 & Urals montane tundra and taiga & 190 & Mesopotamian shrub desert \\
\hline 93 & Central Anatolian steppe & 194 & Red Sea Nubo-Sindian tropical desert and semi-desert \\
\hline 95 & Eastern Anatolian montane steppe & 888 & Northern Temperate Atlantic \\
\hline
\end{tabular}

Tabelle 4 Legende zu den Großeinheiten der Stratification of Europe (EnS), Anzahl der Strata

\begin{tabular}{lcc}
\hline 13 Großeinheiten & Kürzel & Anzahl Strata \\
\hline Alpine North & ALN & 4 \\
Alpine South & ALS & 6 \\
Anatolian & ANA & 2 \\
Atlantic Central & ATC & 5 \\
Atlantic North & ATN & 5 \\
Boreal & BOR & 8 \\
Continental & CON & 12 \\
Lusitanien & LUS & 4 \\
Mediterranean Mountains & MDM & 11 \\
Mediterranean North & MDN & 10 \\
Mediterranean South & MDS & 9 \\
Nemoral & NEM & 5 \\
Pannonian & PAN & 3 \\
\hline
\end{tabular}

(Wascher 2005, Mücher et al. 2006). Für die LANMAP 2 wurden u.a. auch Landnutzungsdaten für die Ableitung und Klassifizierung der Räume hinzugezogen. Daher ist sie, anders als die EnS, nicht für einen Vergleich mit der ELCE geeignet.

Ökogliederung Deutschlands Die Berechnung der landschaftsökologischen Raumgliederung Deutschlands (Schröder et al. 2006) fußt auf demselben methodischen Prinzip wie die Berechnung der ELCE. Folgende Eingangsdaten wurden verwendet: Karte der PNV im Maßstab $1: 2.500 .000$ (Bohn et al. 2000, 2003), Höhe über NN mit einer Auflösung von 30 Bogensekunden (ca. $1 \mathrm{~km} \times 1 \mathrm{~km}$ ) (UNEP Grid), Bodenart aus der BÜK1000 im Maßstab $1: 1.000 .000$ (Bundesanstalt für Geowissenschaften und Rohstoffe - BGR) und Klimadaten des Deutschen Wetterdienstes (DWD) mit einer Auflösung von $1 \mathrm{~km} \times 1 \mathrm{~km}$ 
Tabelle 5 Legende zu den 21 Ökoklassen der Deutschlandgliederung

\begin{tabular}{ll}
\hline ID & Name \\
\hline 8 & Altmark, Prignitz und Uckermark \\
12 & $\begin{array}{l}\text { Hochlagen der kristallinen und paläozoischen } \\
\text { Mittelgebirge }\end{array}$ \\
18 & Brandenburger Jungmoränenlandschaft \\
19 & Leipziger Tieflandsbucht \\
20 & Mecklenburger Seenplatte \\
22 & Ost- und Nordfriesische Marsch \\
26 & Schwäbische Alb \\
30 & Sächsische Börden \\
42 & Niedersächsische Geest, Westfälische Tieflandsbucht \\
43 & Schleswig-Holsteinische Geest und Lüneburger Heide \\
46 & Schleswig-Holsteinische und Mecklenburgische \\
& Jungmoränenlandschaft \\
47 & Niedersächsische Börden, Rheinland und Oberrheintal \\
54 & Allgäu und Hochalpen \\
55 & Alpenvorland \\
56 & Bayerisches Hügelland und Fränkisches \\
& Keuper-Lias-Land \\
57 & Kristalline Mittelgebirge \\
58 & Fränkische Alb \\
62 & Rheinisches Schiefergebirge, südwestdeutsches \\
63 & Schichtstufenland \\
118 & Schwäbisches Schichtstufenland \\
119 & Lausitz und Thüringer Schiefergebirge \\
& Schotterterrassen des Alpenvorlands und \\
\hline & Keuper-Berglands \\
\hline
\end{tabular}

zu Niederschlag, Verdunstung, Temperatur (Monatsmittel Januar bis Dezember für den Zeitraum 1961 bis 1990) sowie zur Globalstrahlung (Monatsmittel März bis November für den Zeitraum 1981 bis 1999). Die in zwei Differenzierungsstufen (73 und 21 Ökoklassen - ÖK), in einer Auflösung von $2 \times 2 \mathrm{~km}^{2}$ vorliegende Raumgliederung diente u. a. der Überprüfung der landschaftsökologischen Repräsentanz deutscher Umweltmonitoringmessnetze (Schröder und Schmidt 2000) und wird als räumliche Bezugsgrundlage in WebGIS-Applikationen verwendet (Kleppin et al. 2008; Pesch et al. 2007). Die Berechnung der Raumgliederung wurde von einem aus Vertretern unterschiedlicher Bundesbehörden zusammengesetzten Arbeitskreis Raumgliederung fachlich begleitet (Schröder et al. 2006). Tabelle 5 zeigt die Legende der Raumgliederung Deutschlands mit 21 Ökoklassen.

\subsection{Methoden}

Der Vergleich der ELCE Gliederung mit den oben beschriebenen Raumgliederungen erfolgte über eine räumliche Verschneidung im GIS. Um eine Vergleichbarkeit zu gewährleisten, wurden für die Gegenüberstellung der ELCE zu den anderen Raumgliederungen ELCE-Versionen mit ähnlich hoher Endklassenanzahl gewählt. Da die Umrisse der Gliederungen nicht deckungsgleich sind, wurden sie räumlich auf ihren Überschneidungsbereich reduziert. Der Vergleich jeweils zweier Raumgliederungen wurde über Kreuztabellen durchgeführt, in denen pro Raumklasse der einen Gliederung die prozentualen Flächenanteile der Raumklassen der anderen Raumgliederung angegeben werden. Der Vergleich zwischen der ELCE einerseits und DMEER, EnS bzw. der Ökogliederung Deutschlands andererseits erfolgte dabei jeweils zweifach: Es wurden sowohl für die EC die Flächenanteile der Raumklassen der zu vergleichenden Raumgliederung bestimmt als auch umgekehrt. Dadurch ließ sich z.B. erkennen, ob die Räume weitgehend kongruent sind oder ob nur kleine Bereiche einer Raumklasse durch die Klassen der anderen Raumgliederung überlagert werden.

Um eine statistische Kenngröße für die Ähnlichkeit der Räume jeweils zweier Raumgliederungen abzuleiten, wurden pro Raumklasse der einen Gliederung die prozentualen Anteile der mindestens $10 \%$ Flächenanteil aufweisenden Raumklassen der anderen Raumgliederung aufsummiert und mit dem Flächenanteil der erstgenannten Raumklasse an der Gesamtfläche gewichtet. Es ergibt sich das sogenannte flächenanteilige Summenprozent. Der minimale Flächenanteil der Raumklassen wurde auf $10 \%$ festgelegt, um kleine Überdeckungsanteile, die weniger als 1/10 der Fläche der betrachteten Raumklasse betragen und damit für die Ermittlung einer Überlagerung unbedeutend sind, von der Berechnung auszuschließen. Die Summe der für alle Raumklassen berechneten flächenanteiligen Summenprozente ergibt einen mittleren Wert für den Überdeckungsgrad der Raumklassen einer Raumgliederung durch die Klassen der Vergleichsraumgliederung.

Zur Veranschaulichung wird im Folgenden die Berechnung der flächenanteiligen Summenprozente und der mittleren Abdeckung am Beispiel von Tabelle 6 beschrieben: Die größte EC der ELCE50, die EC D_19, hat einen Flächenanteil an der Gesamtfläche von 4,3\%. Die DER mit der größten Flächenüberlagerung mit der EC ist die DER 86, die auf 81,4\% der Fläche der EC D_19 vorkommt. Einen weiteren Anteil von mehr als $10 \%$ an der EC D_19 hat die DER 46 mit 18,4\%. Diese Überlagerungsanteile der EC durch die zwei DER betragen zusammen $99,8 \%$. Die EC wird also fast vollständig durch zwei DER überlagert. Multipliziert mit dem Flächenanteil der EC an der Gesamtfläche von 4,3 und geteilt durch 100 ergibt sich ein gewichteter Wert für das flächenanteilige Summenprozent von 4,3\%. Diesen Wert berechnet man auf diese Weise für alle EC und summiert sie auf. Es ergibt sich eine mittlere Abdeckung der EC durch die DER von $84,3 \%$. Bei vollständiger Überdeckung der Klassen durch Vergleichsraumklassen mit einem Flächenanteil von mehr als $10 \%$ läge der Wert für die mittlere Abdeckung bei $100 \%$. 
Tabelle 6 Kreuztabellierung ELCE50 - DMEER; Mindesthäufigkeit 10\% (Auszug)

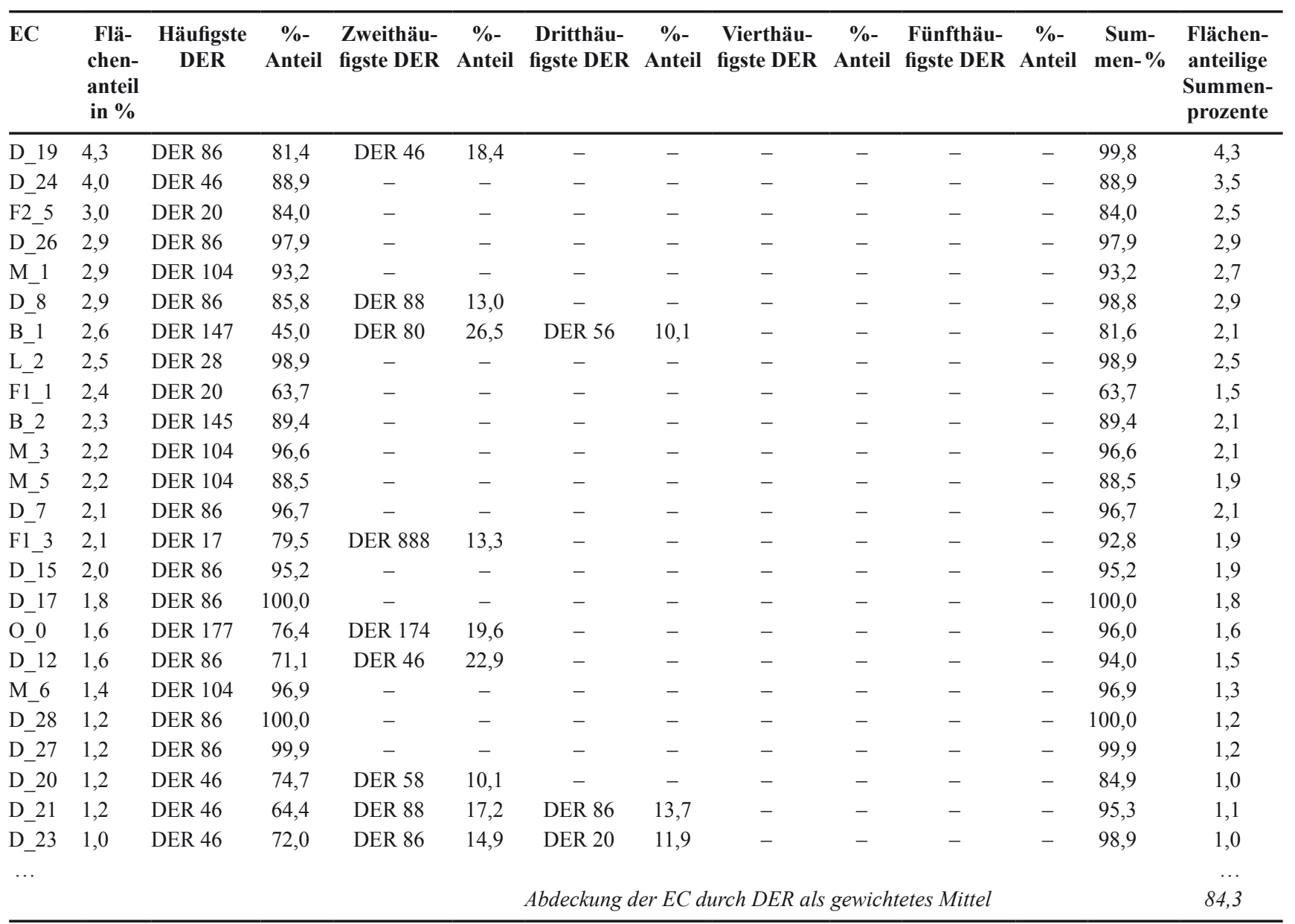

\section{Ergebnisse}

Im Folgenden werden die Ergebnisse der Vergleiche zwischen der ELCE und der DMEER, EnS bzw. der Ökogliederung Deutschlands betrachtet. Eine detaillierte Beschreibung anhand der Kreuztabellen erfolgt dabei ausschließlich für den Vergleich zwischen der ELCE50 und DMEER. Bezüglich der beiden anderen Vergleiche werden nur die wesentlichen Ergebnisse herausgearbeitet.

ELCE50 und DMEER Die Ergebnisse der Flächenverschneidung der ELCE50 und der DMEER werden in der Tabelle 6 (Anteile DER an EC) und der Tabelle 7 (Anteile EC an DER) aufgelistet. Anhand der dort aufgeführten Flächenanteile zeigt sich, dass die EC maximal 4,3\% der Gesamtfläche einnehmen, während die größte DER, die DER 86, 21,6\% der Gesamtfläche abdeckt. Die ELCE50 gliedert kleinere Räume aus, die an verschiedenen Stellen in Europa auftreten können. Die Klassen der DMEER sind hingegen zusammenhängender und z.T. großflächiger in Europa verteilt.

Um einen sinnvollen Vergleich zu gewährleisten, wurde eine ELCE-Version gewählt, die der Klassenanzahl der Ver- gleichsgliederung, in diesem Fall der DMEER, am nächsten kommt. Von den 69 DER der DMEER konnten 52 mit der ELCE50 verschnitten und auf Gemeinsamkeiten und Unterschiede überprüft werden. Bei diesem Vergleich sind 509 von 2.600 (52 DER ×50 EC) möglichen Kombinationen in der Kreuztabelle realisiert. Es lassen sich folgende Auffälligkeiten festhalten:

- Die aufsummierten prozentualen Flächenanteile zeigen eine hohe Abdeckung der EC durch die DER von $84 \%$ (Tabelle 6). Die Abdeckung der DER durch die EC beträgt als gewichtetes Mittel $69 \%$ (Tabelle 7).

- Aufgrund der Verteilung der DMEER in zusammenhängenden, zum Teil sehr großen Flächen haben einzelne DER große Flächenanteile an mehreren EC (Tabelle 6). Abbildung 1 zeigt dies am Beispiel der DER 86 (Scandinavian and Russian taiga), die an 9 EC aus der Hauptformation D einen Anteil von mehr als $71 \%$ hat. Hierbei beträgt der Flächenanteil der DER an sechs der neun EC sogar über $90 \%$ (Tabelle 6). Umgekehrt liegen in der DER 86 drei EC, die einen Anteil von mindestens 10\% aufweisen und insgesamt $41 \%$ der DER abdecken (Ta- 
Tabelle 7 Kreuztabellierung DMEER - ELCE50; Mindesthäufigkeit 10\% (Auszug)

\begin{tabular}{|c|c|c|c|c|c|c|c|c|c|c|c|c|c|}
\hline DER & $\begin{array}{c}\text { Flächen- } \\
\text { anteil } \\
\text { in \% }\end{array}$ & $\begin{array}{c}\text { Häufigste } \\
\text { EC }\end{array}$ & $\begin{array}{c}\%- \\
\text { Anteil }\end{array}$ & $\begin{array}{l}\text { Zweithäu- } \\
\text { figste EC }\end{array}$ & $\begin{array}{c}\%- \\
\text { Anteil }\end{array}$ & $\begin{array}{l}\text { Dritthäu- } \\
\text { figste EC }\end{array}$ & $\begin{array}{c}\%- \\
\text { Anteil }\end{array}$ & $\begin{array}{l}\text { Vierthäu- } \\
\text { figste EC }\end{array}$ & $\begin{array}{c}\%- \\
\text { Anteil }\end{array}$ & $\begin{array}{c}\text { Fünft- } \\
\text { häufigste } \\
\text { EC }\end{array}$ & $\begin{array}{c}\%- \\
\text { Anteil }\end{array}$ & $\begin{array}{c}\text { Sum- } \\
\text { men- \% }\end{array}$ & $\begin{array}{l}\text { Flächenantei- } \\
\text { lige Summen- } \\
\text { prozente }\end{array}$ \\
\hline DER 86 & 21,6 & D_19 & 16,1 & D_26 & 13,3 & D_8 & 11,6 & - & - & - & - & 41,1 & 8,9 \\
\hline DER 104 & 9,2 & $\bar{M}_{-} 1$ & 28,9 & M_3 & 23,2 & M_5 & 20,7 & M_6 & 14,3 & - & - & 87,2 & 8,0 \\
\hline DER 46 & 8,4 & D_24 & 42,2 & D_20 & 10,8 & - & - & - & - & - & - & 53,0 & 4,4 \\
\hline DER 20 & 7,4 & F2_5 & 34,2 & F1_1 & 20,3 & - & - & - & - & - & - & 54,5 & 4,0 \\
\hline DER 28 & 7,3 & L_2 & 34,6 & F2_3 & 25,5 & L_1 & 12,5 & F2_4 & 10,7 & - & - & 83,3 & 6,1 \\
\hline DER 55 & 5,0 & F3_1 & 29,0 & F4_4 & 26,6 & F2_6 & 11,9 & F3_2 & 11,7 & - & - & 79,3 & 3,9 \\
\hline DER 41 & 3,1 & G1_1 & 26,3 & U_2 & 22,4 & F2_6 & 14,8 & - & - & - & - & 63,5 & 2,0 \\
\hline DER 159 & 3,0 & J_2 & 44,2 & J_3 & 44,0 & - & - & - & - & - & - & 88,2 & 2,7 \\
\hline DER 11 & 3,0 & F1_4 & 25,0 & F3_2 & 16,6 & F4_3 & 14,6 & G2_2 & 11,7 & F2_6 & 11,6 & 79,5 & 2,4 \\
\hline DER 145 & 2,7 & B_2 & 77,1 & S_0 & 16,1 & - & - & - & - & - & - & 93,2 & 2,5 \\
\hline DER 12 & 2,6 & G1_2 & 37,5 & G1_1 & 17,5 & U_2 & 11,7 & G2_1 & 10,6 & - & - & 77,4 & 2,0 \\
\hline DER 147 & 2,4 & B_1 & 48,1 & C_1 & 43,2 & - & - & - & - & - & - & 91,3 & 2,2 \\
\hline DER 17 & 2,1 & F1_3 & 77,0 & - & - & - & - & - & - & - & - & 77,5 & 1,6 \\
\hline DER 177 & 1,7 & O_0 & 73,9 & M_1 & 10,2 & - & - & - & - & - & - & 84,2 & 1,4 \\
\hline DER 16 & 1,5 & F5_0 & 47,9 & C_2 & 24,1 & - & - & - & - & - & - & 71,9 & 1,1 \\
\hline$\ldots$ & & & & & & & & & & & & & $\ldots$ \\
\hline \multicolumn{13}{|c|}{ Abdeckung der DER durch EC als gewichtetes Mittel } & 68,9 \\
\hline
\end{tabular}

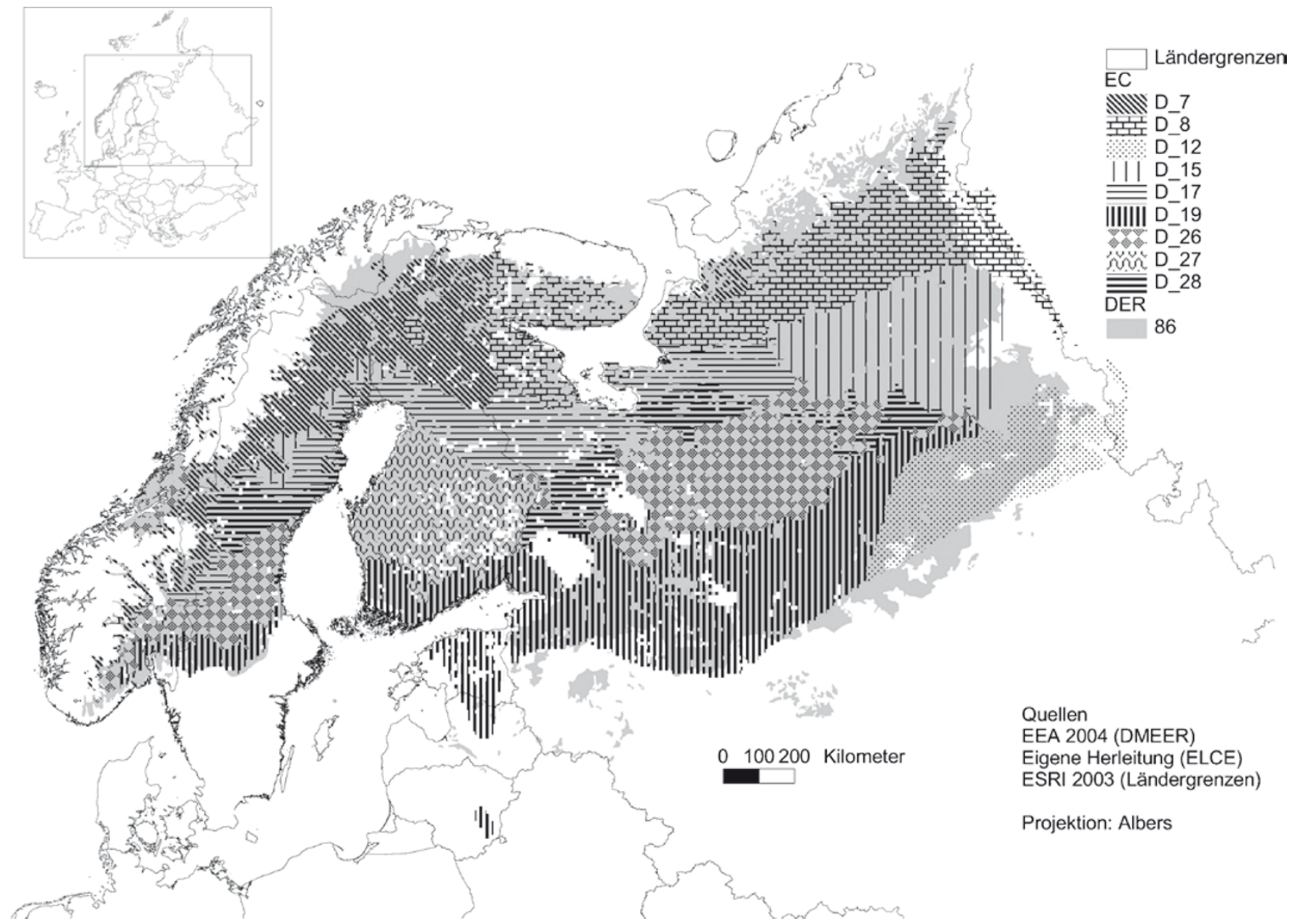

Abb. 1 Überlagerung der DER 86 der DMEER mit 13 EC der ELCE50 
belle 7). Eine ähnliche Tendenz wie DER 86 zeigt die DER 46 (Sarmatic mixed forests). Diese DER hat an vier EC, die aus der PNV-Hauptformation D resultieren, einen Anteil von jeweils mindestens $64 \%$ (Tabelle 6). Umgekehrt beinhaltet die DER 46 zwei EC (D_24, D_20), die mindestens $10 \%$ der Fläche diese DER einnehmen und dabei einen Flächenanteil von insgesamt 53\% ausmachen (Tabelle 7).

- Einige Regionen sind sich räumlich besonders ähnlich: Dies ist zum einen der südliche Bereich der Insel Nowaja Semlia in Nordrussland und der Küstenbereich westlich des Uralgebirges (EC B_2 und DER 145: Northwest Russian/Novaya Zemlya tundra) (Abb. 2) (EC an DER $77 \%$ bzw. DER an EC 89\% Anteil), außerdem große Teile Großbritanniens und Nordirlands (EC F1_3 und DER 17: Celtic broadleaf forests) (EC an DER 77\% bzw. DER an EC 80\%) und als drittes die Kaspische Senke (EC O_0 und DER 177: Caspian Lowland desert) (EC an DER $74 \%$ bzw. DER an EC 76\%). Hohe, wenn auch nicht ganz so ausgeprägte Übereinstimmungen zeigen die EC F5_0 (Kaukasus-Region) und die DER 16 ,Caucasus mixed forests“ (EC an DER $48 \%$ bzw. DER an
EC $68 \%$ ). Im Bereich der DER 147 „Scandinavian montane birch forests and grasslands" und der EC B_1 gibt es gegenseitig Übereinstimmungen von fast $50 \%$ (EC an DER $48 \%$ bzw. DER an EC 45\%). Im Fall der DER 20 „Central European Mixed Forests“, die hauptsächlich in Polen, Weißrussland und der Ukraine vorkommt und 7\% der Fläche der DMEER einnimmt, decken die EC F2_5 (34\%) und F1_1 20\% zusammen über 50\% der DER ab (Tabelle 7). Die DER 20 hat ihrerseits einen Anteil von $84 \%$ an der EC F2_5, und einen Anteil von $64 \%$ an der F1_1 (Tabelle 6).

ELCE100 und EnS Ausgenommen derjenigen EnS-Strata, die nur in Nordafrika und der Türkei vorliegen, stehen für den Vergleich noch 80 Strata zur Verfügung. Für die Verschneidung wurde die ELCE100 ausgewählt, von der auf den von der EnS festgelegten Ausschnitt 80 EC entfallen. Von den insgesamt 6.400 (80 Strata $\times 80$ EC) möglichen Kombinationen sind 1.091 Kombinationen realisiert. Die Kreuztabellierung ergibt eine Abdeckung der EC durch die Strata von $67 \%$ als gewichtetes Mittel, während die Abdeckung der Strata durch die EC $72 \%$ beträgt. Abbildung 3 zeigt drei Fälle, in denen sowohl der Anteil einer EC an ei-
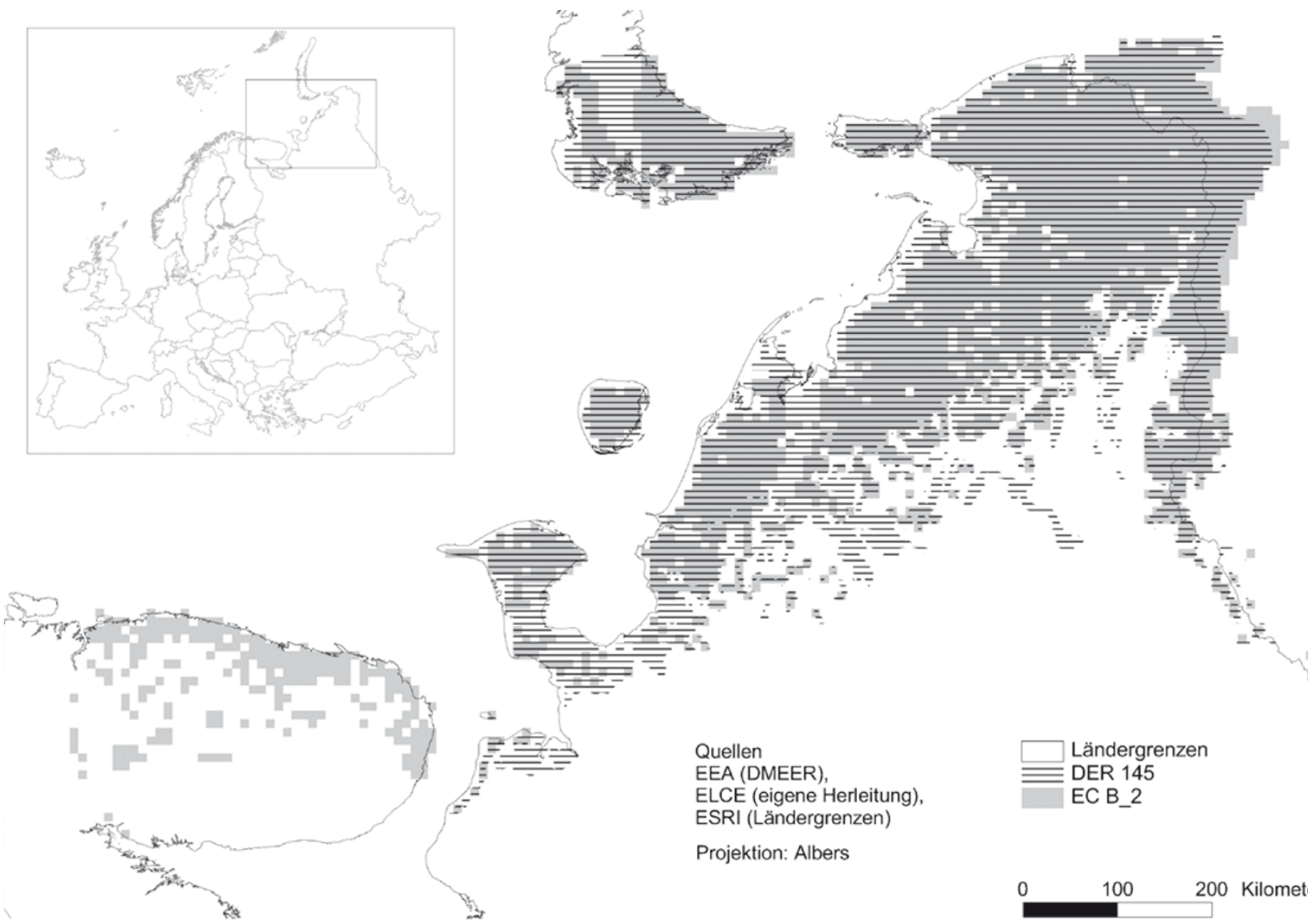

Quellen

EEA (DMEER),

ELCE (eigene Herleitung),

ESRI (Ländergrenzen)

Projektion: Albers

Abb. 2 Überlagerung der EC B_2 der ELCE50 mit der DER 145 der DMEER 


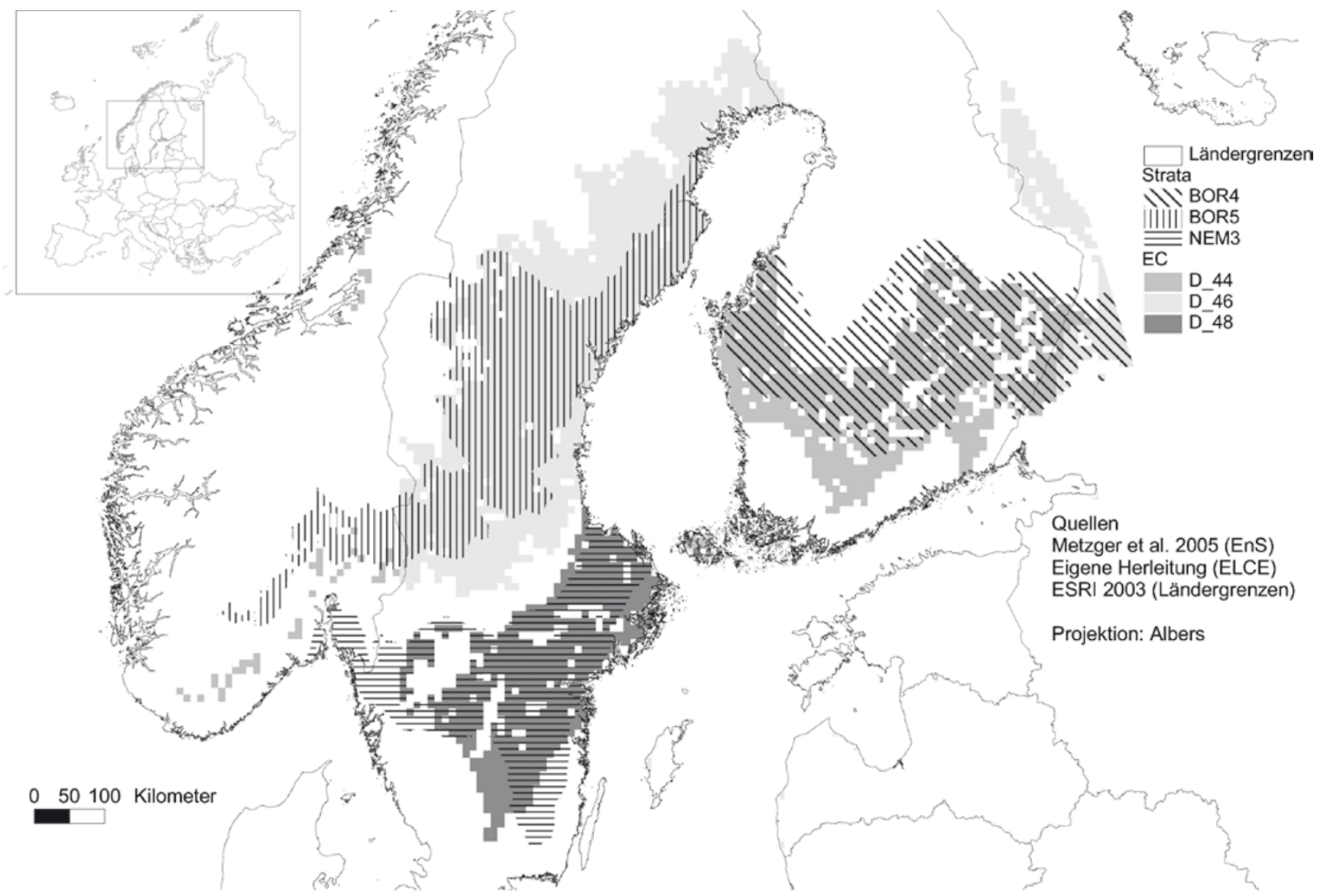

Abb. 3 Überlagerung der EC D_44, D_46 und D_48 der ELCE100 mit den Strata BOR4, BOR5 und NEM3 der Stratification of Europe

nem Stratum als auch der Anteil des Stratums an der EC hoch ist: Bei der EC D 44 und dem Stratum BOR4 (in Mittel- und Südfinnland, Nordwest-Russland) (EC an Stratum $77 \%$, Stratum an EC 67\%), der EC D_48 und dem Stratum NEM3 (Provinz Oestfold in Südnorwegen, Süden Schwedens) (EC an Stratum 70\%, Stratum an EC $75 \%$ ) sowie bei der D_46 und dem Stratum BOR5 (Streifen von Südnorwegen bis Mittel-Schweden) (EC an Stratum 46\% bzw. Stratum an EC $81 \%$ ).

ELCE150 und Ökogliederung Deutschlands Die Raumgliederung Deutschlands in 21 Klassen (ÖK) wird der ELCE150 gegenübergestellt. Bei dieser ELCE-Version entfallen 21 EC auf den Ausschnitt der BRD. Von demnach insgesamt $441(21 \times 21)$ Kombinationsmöglichkeiten sind 170 Kombinationen realisiert. Die Kreuztabellierung ergibt eine Abdeckung der EC mit den ÖK von insgesamt $75 \%$, während die ÖK für die Überdeckung mit EC einen Wert von $80 \%$ aufweisen. Abbildung 4 zeigt anhand von Norddeutschland die Übereinstimmungen der ELCE und der Raumgliederung Deutschlands im östlichen Hügelland Schleswig-Holsteins und dem nördlichen Teil MecklenburgVorpommerns (EC F4_1, ÖK 46; ÖK an EC 55\%, EC an
ÖK 74\%) sowie in den nord- und ostfriesischen Marschgebieten (EC U 8, ÖK 22; ÖK an EC 65\%, EC an ÖK 90\%). Deutliche Überschneidungen gibt es weiterhin im Bereich des Rheinischen Schiefergebirges und der südwestdeutschen Schichtstufenlandschaft (EC F3_10, ÖK 62; ÖK an EC 79\%; EC an ÖK 48\%). Der Bereich Süd- und Mitteldeutschlands mit den Höhenzügen der Schwäbischen Alb (ÖK 26) und der Fränkischen Alb (ÖK 58) ist bei der ELCE nicht so differenziert ausgegliedert. Eine gute Übereinstimmung liegt wiederum beim Alpenvorland (ÖK 55) und im Allgäu und den Hochalpen (ÖK 54) vor. Die ÖK 54 hat an EC F4_13 einen Anteil von 81\% (EC an ÖK 39\%) und an der EC F4_14 63\% (EC an ÖK 43\%), während die ÖK 55 an F4 14 noch $37 \%$ einnimmt (EC an ÖK 24\%).

Exemplarisch wurde für die ÖK 46 und die EC F4_1 geprüft, ob die hohe flächenmäßige Übereinstimmung auch im Hinblick auf die ökologischen Eigenschaften bestätigt werden kann. Dem Vergleich liegt dabei die Beschreibung der ÖK 46 von Schröder et al. (2006) sowie die statistische Auswertung der Eigenschaften der EC F4_1 anhand der für die Ableitung der ELCE verwendeten Daten zugrunde. Im Hinblick auf die PNV würden sich in beiden Raumklassen 


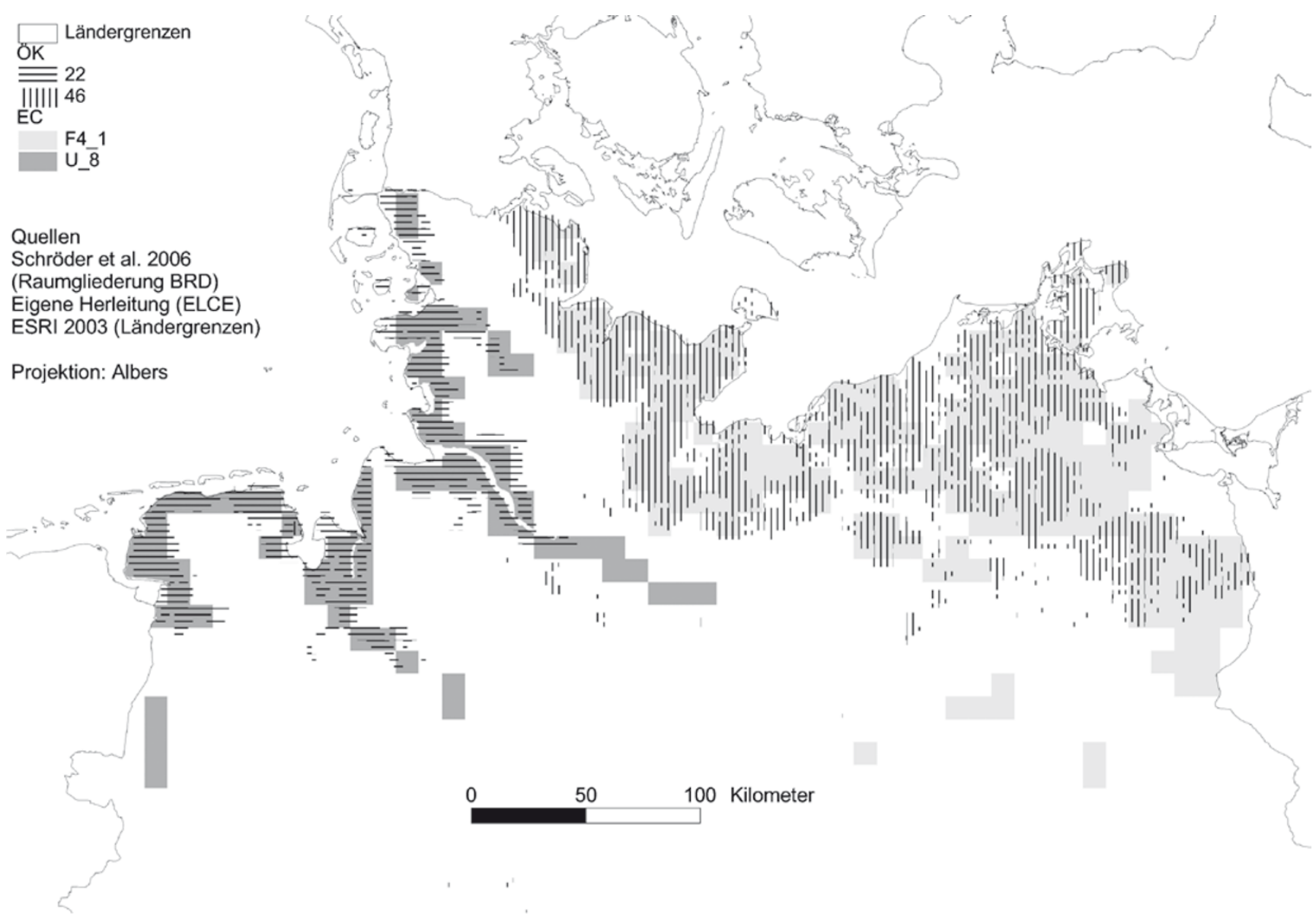

Abb. 4 Überlagerung der EC F4_1 und U_8 der ELCE100 mit den ÖK 22 und 46 der Raumgliederung Deutschland (Ausschnitt Norddeutschland)

unter natürlichen Bedingungen größtenteils Buchenwälder z.T. mit Eichen entwickeln. In Bezug auf die Bodenart liegen der ÖK genauere Informationen zugrunde. Während die EC F4_1 als häufigste Bodenart den reinen Schluff ausweist, sind in der ÖK 46 lehmige Sande bis Lehme vorherrschend. Die Niederschläge werden bei Schröder et al. (2006) in Bezug auf die Werte für Deutschland als ganzjährig bis zu $15 \mathrm{~mm}$ niedriger beschrieben. Für Deutschland werden bei Schröder et al. (2006) Niederschlagsmediane für Januar und Juli von 56 bzw. $72 \mathrm{~mm}$ angegeben. Die Werte der EC F4_1 für diese Monate liegen mit 49 und $62 \mathrm{~mm}$ ebenfalls niedriger.

\section{Diskussion}

Vergleich ELCE - DMEER Im Hinblick auf die den beiden Raumgliederungen zugrunde liegende methodische Vorgehensweise zeigen beide Ansätze Gemeinsamkeiten. Während für die ELCE ein Entscheidungsbaumverfahren angewendet wurde, erfolgte die Berechnung der DMEER mithilfe eines hierarchischen Clusterverfahrens. Dennoch war die Ableitung der DMEER nicht vollständig bearbeiterunabhängig, da das erste Klassifikationsergebnis auf Basis von Expertenwissen entstand und nachträglich verändert wurde. Die ITE-Gliederung von Bunce (1995), die neben der PNV-Karte Europas Eingang in die Ableitung der DMEER hatte, stellt mit einer Auflösung von $0,5 \times 0,5$ Bogenminuten $\left(\right.$ ca. $56 \times 56 \mathrm{~km}^{2}$ ) für die DMEER eine gering aufgelöste Grundlage dar.

Der Unterschied in den zwei Gesamtwerten für die Überdeckung zwischen den DER und den EC (EC durch DER $84 \%$, DER durch EC $69 \%$ ) liegt darin begründet, dass viele EC durch ihre starke Ausdifferenzierung einen kleinen Anteil an den DER einnehmen. Letztere betragen häufig weniger als $10 \%$ der Gesamtfläche der jeweiligen Raumklasse und gehen somit nicht in die Berechnung des gewichteten Mittels ein. Die verschiedenen DER hingegen haben an den kleinen EC jeweils einen hohen Anteil. Selbst wenn man das gewichtete Mittel nur für die häufigste DER an den EC ermittelt, beträgt der Überdeckungsgrad noch $66 \%$.

Räumlich differenziert betrachtet zeigen die ELCE50 und die DMEER Übereinstimmungen, was auf die gemein- 
same Verwendung der PNV als Berechnungsgrundlage zurückzuführen ist. Allerdings existieren auch Unterschiede. Bei der DMEER liegt beispielsweise keine derart deutliche Ausdifferenzierung der PNV-Hauptformation D vor wie bei der ELCE50. Die DMEER bildet weiterhin räumlich enger zusammenhängende Raumklassen $\mathrm{ab}$, als dies bei der ELCE50 der Fall ist. Letztere weist z.B. Raumklassen auf, die sowohl im Westen als auch im Osten Europas vorkommen. Dies lässt sich darauf zurückführen, dass im Gegensatz zur DMEER bei der ELCE auf eine Nachbearbeitung der Raumklassengeometrien verzichtet wurde. Dies kann als Vorteil der ELCE in Bezug auf deren Anwendung zur Optimierung von Umweltmessnetzen betrachtet werden. Es ist durchaus sinnvoll, im Rahmen der Kostenreduktion z. B. für ein europäisches Bodendauerbeobachtungsmessnetz auch weit entfernte Gebiete als zu einen Raum gehörig zu betrachten, wenn sie mit denselben ökologischen Bedingungen ausgestattet sind.

Vergleich ELCE - EnS Obwohl der EnS keine Daten zur PNV zugrunde liegen, zeigen sich in der Gesamtheit dennoch gute Übereinstimmungen zwischen beiden Raumgliederungen. Trotzdem sind beide durch die unterschiedlichen Berechnungssysteme und Eingangsdaten jeweils eigenständige Gliederungen, die je nach Anwendungszweck geeignete Grundlagen darstellen. Ein Grund für die Unterschiede ist, dass keine geeigneten Bodendaten für die Ableitung der EnS zur Verfügung standen. Zudem ist die EnS in Südeuropa wesentlich differenzierter als in Nordeuropa, während die ELCE durch eine flächenproportionale Verteilung der Endklassen auf die PNV-Gebiete in Mitteleuropa besonders stark untergliedert ist (Hornsmann et al. 2008, Weustermann et al. 2009). Die EnS wurde für Nord- und Südeuropa getrennt berechnet, wobei für Südeuropa mit 30\% der Fläche 30 Strata entfallen, für Nordeuropa jedoch bei $70 \%$ der Fläche 40 Strata. Metzger et al. (2005) begründen ihr Vorgehen mit den Erfahrungen aus der Vorgängerklassifizierung von Bunce et al. (1996a) und einer ersten Clusterung, bei der Südeuropa stark differenziert wurde und nur sehr wenige Klassen auf Nordeuropa entfielen. Dieses Vorgehen stellt einen Bearbeitereingriff in die Klassifikation dar.

Bei der Ableitung der EnS wurden die PNV nicht einbezogen: Metzger et al. (2005) sahen vielmehr das Klima als wichtigste Eingangsgröße für die Ableitung einer ökologischen Raumgliederung. Im Gegensatz zu den 46 Klimaeingangskarten der ELCE, die den Jahresgang der monatlichen Mittelwerte von vier Klimamerkmalen (Temperatur, Niederschlag, relative Luftfeuchtigkeit und Sonnenscheindauer) in die Analyse einbeziehen, wurde für die EnS eine Auswahl von je vier Monaten (Januar, April, Juli und Oktober) von drei Klimaparametern (Temperatur, Niederschlag und Sonnenscheindauer) getroffen. Diese Auswahl war laut Metzger et al. (2005) nötig, um den Rechenaufwand zu reduzieren und basiert auf einer statistischen Analyse, die bei der Vorgängerversion von Bunce et al. (1996b) durchgeführt wurde.

Bedingt durch die räumliche Begrenzung der zugrunde liegenden Klimadaten, umfasst die EnS nicht den gesamten europäischen Raum. Die 16 Klimainformationen liegen in einer Auflösung von $10 \times 10$ Bogenminuten $\left(\right.$ ca. $\left.19 \times 19 \mathrm{~km}^{2}\right)$ vor. Sie rechtfertigen daher die hohe Auflösung der EnS in $1 \times 1 \mathrm{~km}^{2}$ auf den ersten Blick nicht. Metzger et al. (2005) rechtfertigt die Wahl der Auflösung damit, dass die Ausprägung von ökologischen Strukturen auf lokaler Ebene relativ unabhängig von großräumigen Klimadaten ist, sondern dass die Topografie diese Strukturen prägt. Diese lag in Form eines gerasterte Höhenkarte in der Auflösung von $1 \times 1 \mathrm{~km}^{2}$ vor (Metzger et al. 2005).

Vergleich ELCE - Ökogliederung Deutschlands Der Vergleich zur höher aufgelösten landschaftsökologischen Raumgliederung Deutschland hat gezeigt, dass die ELCE einige auf nationaler Ebene identifizierte homogene Raumeinheiten ebenfalls ausgliedert. Aufgrund der gröber aufgelösten Eingangsdaten stellt die ELCE jedoch für nationale Aufgaben keine Alternative zur existierenden Raumgliederung Deutschlands dar. Gleiches lässt sich vermutlich auch im Hinblick auf andere nationale Raumgliederungen feststellen.

\section{Schlussfolgerungen}

Zusammenfassend können folgende wesentliche Unterschiede der ELCE zu den anderen Gliederungen Europas, der DMEER und der EnS, genannt werden: Die Eingangsdaten der ELCE sind insbesondere im Hinblick auf das Klima umfassender als bei den anderen Gliederungen. Zudem ist das Entscheidungsbaumverfahren CART sehr gut nachvollziehbar und anschaulich. Im Ergebnis sind die Räume der ELCE zum großen Teil differenzierter, d.h. kleiner und stärker unterteilt, sie kommen zudem an mehreren Orten in Europa vor. Es hat keine Nachbearbeitung der Ergebnisraumgliederungen stattgefunden. Je nach Anwendungsfall kann auf ELCE-Versionen mit 40 bis 200 Klassen zurückgegriffen werden.

Trotz dieser Unterschiede konnten auch einige Übereinstimmungen in den Raumgliederungsergebnissen gefunden werden.

Anhand des statistischen sowie inhaltlichen Vergleichs mit den ausgewählten Raumgliederungen verschiedener ELCE-Versionen kann die ELCE als fachlich plausibel und als geeignete Ergänzung zu den europäischen Vergleichsgliederungen eingestuft werden. Sie kann jedoch kein Ersatz für nationale Gliederungen sein, die auf höher aufgelösten Daten basieren. Aufgrund der Ergebnisse dieses Vergleichs und der im zweiten Artikel der Serie (Weustermann et al. 2009) beschriebenen Erkenntnisse zur Qualität der Gliede- 
rung stellt die ELCE für europaweite Repräsentanzanalysen von Messnetzen eine geeignete Grundlage dar.

\section{Empfehlungen}

Um den Einfluss der PNV-Hauptformationen auf die Einteilung der ELCE zu reduzieren (Weustermann et al. 2009), empfiehlt sich eine Neuberechnung der ELCE. Diese verbesserte, unabhängigere Ableitung kann nur dann durchgeführt werden, wenn mehr als 64 Ausprägungen einer Zielvariablen in den CART-Berechnungen zugrunde gelegt werden können. In Anbetracht der Klimaveränderung in den vergangenen 20 Jahren wäre es zudem sinnvoll, der Ableitung einer Raumgliederung für Europa aktuellere Klimadaten zu verwenden, sobald diese in geeigneter Form für Europa vorliegen. In Ergänzung zu der landschaftsökologischen Raumgliederung Deutschlands sollte die ELCE in zukünftigen Arbeiten auch mit anderen nationalen Raumgliederungen verglichen werden.

\section{Ausblick}

Im folgenden Artikel der Serie steht die Anwendung der Raumgliederung für die Repräsentanzanalyse ausgewählter europaweiter Umweltmonitoringmessnetze im Mittelpunkt. Anhand der Berechnung der häufigkeitsstatistischen Landschaftsrepräsentanz kann mithilfe der ELCE ermittelt werden, inwieweit sich Messstellen eines ausgewählten Umweltbeobachtungsprogramms flächenproportional auf die landschaftsökologischen Raumeinheiten verteilen. Dies kann wichtige Hinweise dafür liefern, in welchen Raumeinheiten es sinnvoll ist, Messnetze gezielt auszudünnen oder zu ergänzen.

\section{Literatur}

Bohn U, Neuhäusl R, Gollub G; Hettwer C, Neuhäuslová Z, Schlüter H, Weber H (2000) Karte der natürlichen Vegetation Europas/Map of the natural vegetation of Europe, Maßstab/scale 1:2.500.000. Teil 1, Erläuterungstext mit CD-ROM. Teil 2, Legende. Teil 3, Karten. Landwirtschaftsverlag, Münster

Bohn U, Neuhäusl R, Gollub G; Hettwer C, Neuhäuslová Z, Schlüter H, Weber H (2003) Karte der natürlichen Vegetation Europas/Map of the natural vegetation of Europe, Maßstab/scale 1:2.500.000. Teil 1, Erläuterungstext mit CD-ROM. Teil 2, Legende. Teil 3, Karten. Landwirtschaftsverlag, Münster

Bunce RGH (1995) A European land classification. Institute of Terrestrial Ecology, Merlewood

Bunce RGH, Barr, CJ, Clarke RT, Howard DC, Lane AMJ (1996a) Land classification for strategic ecological survey. J Environ Manage 47:37-60

Bunce, RGH, Watkins, J W, Brignall, P,.Orr, J (1996b) A comparison of the environmental variability within the European Union. In: Jongman RHD (ed) (1996) Ecological and landscape consequen- ces of landuse change in Europe. European Centre for Nature Conservation, Tilburg, pp 82-90

CRU (2002) Global climate dataset CRU average climatology (CL) 2.0. http://www.cru.uea.ac.uk/cru/data/hrg.htm (Stand: 28. Oktober 2008)

CRU (2008) High-resolution gridded dataset. CRU time-series (TS) 1.2. http://www.cru.uea.ac.uk/ timm/grid/CRU_TS_1_2.html (Stand: 28. Oktober 2008)

European Environment Agency (EEA) dataservice (2004) http://dataservice.eea.eu.int/dataservice/ (Stand: 28. Oktober 2008)

ESRI (2003) ed. Data and maps. Redlands, CA, USA

ETC/NC (1997) ed. The Digitized Map of European Ecological Regions (DMEER). A tool for the assessment of biodiversity in Europe. Paris (Final Draft)

FAO (1996) The digitized soil map of the world including derived soil properties. FAO, Rome

Hastings DA, Dunbar PK, Elphingstone GM, Bootz M, Murakami H, Maruyama H, Masaharu H, Holland P, Payne J, Bryant NA, Logan TL, Muller J-P, Schreier G, MacDonald JS (eds) (1999) The Global Land One-kilometer Base Elevation (GLOBE) digital elevation model, Version 1.0. National Oceanic and Atmospheric Administration, National Geophysical Data Center, USA Digital data base on the World Wide Web http://www.ngdc.noaa.gov/ mgg/topo/globe.html (Stand: 28. Oktober 2008)

Hornsmann I, Schmidt G, Schröder W (2008) Berechnung einer landschaftsökologischen Raumgliederung Europas. Umweltwiss Schadstoff Forsch 20:25-35

Jongman RHG, Bunce RGH, Metzger MJ, Mücher CA, Howard DC, Mateus VL (2006) Objectives and applications of a statistical environmental stratification of Europe. Landsc Ecol 21:409-419

Kleppin L, Schröder W, Pesch R, Schmidt W (2008) Entwicklung und Erprobung einer Metadaten- und WebGIS-Applikation für das Expositionsmonitoring mit Moosen in Deutschland. Umweltwiss Schadstoff Forsch 20:38-48

Metzger MJ, Bunce RGH, Jongman RHG, Mücher CA, Watkins JW (2005) A climatic stratification of the environment of Europe. Global Ecol Biogeogr 14:549-563

Mitchell TD, Carter TR, Jones PD, Hulme M, New M (2004) A comprehensive set of high-resolution grids of monthly climate for Europe and the globe: the observed record (1901-2000) and 16 scenarios (2001-2100), Tyndall Centre Working Paper no. 55. Tyndall Centre for Climate Change Research, University of East Anglia, Norwich, UK

Mücher CA, Wascher DM, Klijn JA, Koomen AJM, Jongman RHG (2006) A new European Landscape Map as an integrative framework for landscape character assessment. In: Bunce RGH, Jongman RHG (eds) Landscape Ecology in the Mediterranean: inside and outside approaches. Proceedings of the European IALE Conference 29 March - 2 April 2005, Faro, Portugal. IALE Publication Series 3:233-243

New M, Lister D, Hulme M, Makin I (2002) A high-resolution data set of surface climate over global land areas. Clim Res 21:1-25

Painho M, Augusto G (2005) A Digital Map of European Ecological Regions. In: Bohn U, Hettwer C, Gollub G. (eds) Anwendung und Auswertung der Karte der natürlichen Vegetation Europas/ Application and Analysis of the Map of the Natural Vegetation of Europe. BfN-Scripten 156:27-36

Pesch R, Schmidt G, Schröder W, Aden C, Kleppin L, Holy M (2007) Development, implementation and application of the WebGIS MossMet. In: Tochtermann K, Scharl A (eds) The Geospatial Web. How geo-browsers, social software and the Web 2.0 are shaping the network society. Springer, London, pp 191-200

Schmidt G, Aden, C, Kleppin L, Pesch R, Schröder W (2009): Integration of long-term environmental data by the example of the UNECE Heavy Metals in Mosses Survey in Germany: Application of 
a WebGIS-based metadata system. In: Müller F, Klotz S, Schubert H (eds) Long-Term Ecological Research - Between Theory and Application. Springer, Berlin (in press)

Schmidt G, Schröder W (2008) Ökologische Raumgliederung Europas - Vorwort. Umweltwiss Schadstoff Forsch 20:23-24

Schröder W, Schmidt G (2000) Raumgliederung für die Ökologische Umweltbeobachtung des Bundes und der Länder. Umweltwiss Schadstoff Forsch 12:237-243

Schröder W, Schmidt G, Hornsmann I (2006) Landschaftsökologische Raumgliederung Deutschlands. In: Fränzle O, Müller F, Schröder W (Hrsg) Handbuch der Umweltwissenschaften. Grundlagen und Anwendungen der Ökosystemforschung. Ecomed Verlag, Landsberg am Lech München Zürich, Kap. V-1.9, 17. Erg.Lfg. $1-101$
USGS (2008) HYDRO1k Elevation Derivative Database http://eros. usgs.gov/products/elevation/gtopo30/hydro/index.html (Stand: 25. September 2008)

Wascher DM (ed) (2005) European landscape character areas. typlogies, cartography and indicators for the assessment of sustainable landscapes. Final project report as deliverable from the EU's accompanying measure project European Landscape Character Assessment Initiative (ELCAI), funded under the 5th Framework Programme on Energy, Environment and Sustainable Development (4.2.2). Alterra Report No. 1254. http://www.landscape-europe.net/ELCAI projectreport book_amended.pdf (Stand: 20. Oktober 2008)

Weustermann I, Schmidt G, Schröder W (2009) Beschreibung der landschaftsökologischen Raumgliederung Europas - Teil 2. Umweltwiss Schadstoff Forsch 21:94-109 